\title{
Neurophotonics
}

Neurophotonics.SPIEDigitalLibrary.org

\section{Photoacoustic brain imaging: from microscopic to macroscopic scales}

Junjie Yao

Lihong V. Wang 


\title{
Photoacoustic brain imaging: from microscopic to macroscopic scales
}

\author{
Junjie Yao and Lihong V. Wang* \\ Washington University in St. Louis, Department of Biomedical Engineering, Optical Imaging Laboratory, One Brookings Drive, \\ St. Louis, Missouri 63130
}

\begin{abstract}
Human brain mapping has become one of the most exciting contemporary research areas, with major breakthroughs expected in the coming decades. Modern brain imaging techniques have allowed neuroscientists to gather a wealth of anatomic and functional information about the brain. Among these techniques, by virtue of its rich optical absorption contrast, high spatial and temporal resolutions, and deep penetration, photoacoustic tomography (PAT) has attracted more and more attention, and is playing an increasingly important role in brain studies. In particular, PAT complements other brain imaging modalities by providing high-resolution functional and metabolic imaging. More importantly, PAT's unique scalability enables scrutinizing the brain at both microscopic and macroscopic scales, using the same imaging contrast. In this review, we present the state-of-the-art PAT techniques for brain imaging, summarize representative neuroscience applications, outline the technical challenges in translating PAT to human brain imaging, and envision potential technological deliverables. $\odot$ The Authors. Published by SPIE under a Creative Commons Attribution 3.0 Unported License. Distribution or reproduction of this work in whole or in part requires full attribution of the original publication, including its DOI. [DOI: 10.1117/1.NPh.1.1.011003]
\end{abstract}

Keywords: photoacoustic tomography; photoacoustic microscopy; optical absorption contrast; deep brain imaging; functional imaging; molecular imaging; metabolic imaging.

Paper 14018VSSR received Feb. 27, 2014; revised manuscript received Mar. 28, 2014; accepted for publication Mar. 31, 2014; published online May 28, 2014.

\section{Introduction}

Although scientists have been avidly discovering the secrets of the brain, the knowledge accumulated so far still falls far short of a comprehensive understanding. ${ }^{1,2}$ Due to modern biomedical technologies, especially imaging technologies, our understanding of the brain has advanced over the last few decades at an accelerating speed. ${ }^{3,4}$ Looking back, the history of neuroscience is also a history of applying new imaging technologies to look at the brain in a more informative way: finer resolution, higher speed, deeper penetration, and new functionality. Given its impact, brain research is, understandably, the most attractive and rewarding application of newly developed imaging technologies.

So far, a number of imaging technologies have been applied in neuroscience studies. ${ }^{3}$ Among them, two-photon microscopy and functional magnetic resonance imaging (fMRI) are the major tools of choice. At the microscopic scale, two-photon microscopy allows detailed and direct examination of neuroactivities in small animal models, such as action potential firing, the release of signaling molecules, and extra-cellular ion pumping. ${ }^{5}$ At the macroscopic scale, fMRI has been extensively used to map human brain activities, allowing indirect coarse-grained localization of complex cognitive functions, such as language, emotion, decision-making, learning, and memory. ${ }^{6}$ Collectively, modern brain imaging technologies have provided a solid foundation and the strong springboard needed for the next advance of neuroscience.

Naturally, it is necessary to relate two-photon microscopic findings on small animals to the macroscopic fMRI observations on humans. ${ }^{3}$ However, correlation between the two imaging

*Address all correspondence to: Lihong V. Wang, E-mail: Ihwang@ wustl.edu scales is truly an engineering challenge, because two-photon microscopy cannot provide penetration into human brain through an intact skull, ${ }^{7}$ and fMRI cannot achieve spatial resolution at the single cell level. ${ }^{3}$ Beyond the resolution discrepancy, an even more fundamental constraint is the dramatically different signal contrasts of the two modalities. In particular, the relationship between fMRI signals and underlying brain activities remains unclear. ${ }^{3}$ Therefore, there is a gap between the macroscopic activity patterns available in humans and the microscopic activity details available in small animal models. Bridging this gap provides a unique opportunity for photoacoustic tomography (PAT), a hybrid imaging modality that can readily encompass both the microscopic and macroscopic worlds with the same contrast mechanism. ${ }^{8-10}$

Briefly, PAT is based on the photoacoustic (PA) effect, which starts with optical absorption by tissue molecules and ends with ultrasonic emission through thermoelastic expansion. The combination of optical excitation with ultrasonic detection immediately offers PAT two advantages: (1) the resultant PA signal is sensitive to the rich optical absorption contrast of the biological tissue, which provides the functional and metabolic imaging capabilities and (2) the acoustic waves are not as sensitive to scattering as light, which leads to high spatial resolution in deep tissue. Detailed reviews of PAT technologies can be found in several recent publications. ${ }^{9,11,12}$

In this review, we will present the state-of-the-art PAT technology, focusing on its multiscale, functional, and metabolic brain imaging capabilities. Then, we will summarize the representative applications of PAT in neuroscience studies. Further improvements in PAT of the brain, especially for humans, will be discussed. The potential paths toward clinical brain imaging by PAT will be outlined, and likely achievements in the coming years will be envisioned. 


\section{Multiscale PAT of the Brain}

The brain is a multiscale, multilayer, and multicompartment system for robust behaviors. Although a human brain has an average volume of $\sim 1450 \mathrm{~cm}^{3}$, single neurons and glial cells, the building blocks of the brain, are on the scale of micrometers. ${ }^{13}$ Although the total human cortex is about 3-mm thick, the thickness of each of the six cortical layers is on the scale of a few hundreds of micrometers. Through different implementations, PAT is capable of imaging the brain at different length scales. The spatial resolution of PAT can be tuned in both the optical and acoustic domains, while the imaging depth changes accordingly. ${ }^{9}$ The optimum tradeoff between spatial resolution and imaging depth depends on the specific application. As a rule of thumb, the ratio of the imaging depth to the spatial resolution of different PAT implementations is $\sim 200$, qualifying PAT as a high-resolution imaging modality across a wide length scale range. ${ }^{9}$

Although different classifications of PAT exist, we classify it based on the imaging depth in units of the optical transport mean free path (TMFP, $\sim 1 \mathrm{~mm}$ in muscle and $\sim 0.6 \mathrm{~mm}$ in the brain). ${ }^{7}$ This classification emphasizes PAT's tolerance of optical scattering, a primary limiting factor in high-resolution optical imaging. Based on the targeted imaging depth, PAT falls into three groups: (quasi)ballistic PAT, with an imaging depth smaller than one TMFP (typically $<0.6 \mathrm{~mm}$ ); quasidiffusive PAT, with an imaging depth of 1 to 10 TMFPs (typically 0.6 to $6 \mathrm{~mm}$ ); and diffusive PAT, with an imaging depth larger than 10 TMFPs (typically $>6 \mathrm{~mm}$ ). For each depth regime, we will discuss the relevant PAT technologies, their imaging characteristics and representative applications in neuroscience.

\section{1 (Quasi)ballistic PA Imaging of the Brain}

Ballistic PAT targets the imaging depth of one TMFP, which is similar to the thickness of a mouse cortex. Ideally, the term "ballistic imaging" is based on unscattered photons. In reality, morescattered quasiballistic photons are also used to increase the signal strength. For brevity, subsequent use of "ballistic" in this review also refers to "quasiballistic" unless otherwise noted. In the ballistic regime, the representative PAT implementation is optical-resolution photoacoustic microscopy (OR-PAM). ${ }^{14,15}$

As shown in Fig. 1(a), in OR-PAM, the excitation laser beam is tightly focused by an objective lens to an optical-diffractionlimited spot, and a single-element ultrasonic transducer detects the resultant PA signals. Although the ultrasonic transducer is often confocally aligned with the objective lens for optimum detection sensitivity, the optical focusing is generally more than 10 times tighter by diameter than the acoustic focusing, and thus the lateral resolution of OR-PAM is determined by the optical focusing. The axial resolution of OR-PAM is determined by the detection bandwidth of the ultrasonic transducer, (a)

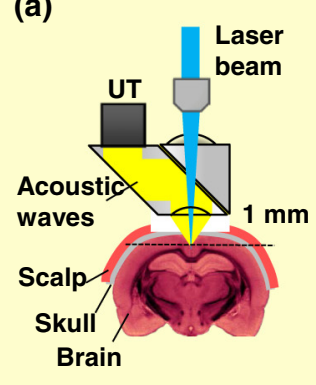

OR-PAM (mouse)

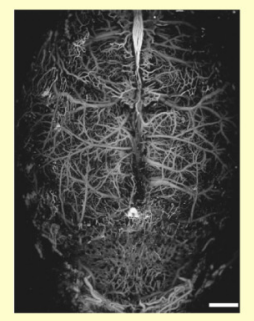

(b)
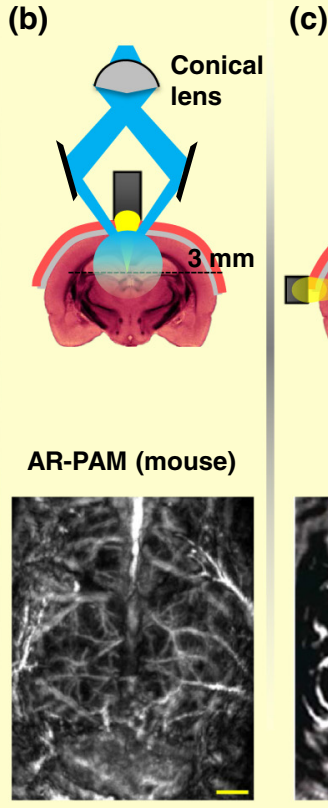

(c)

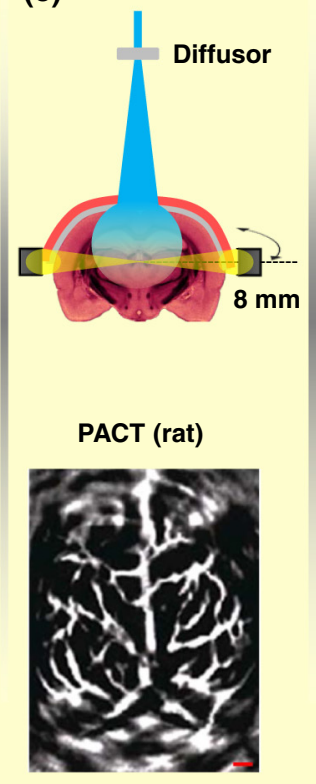

(d)
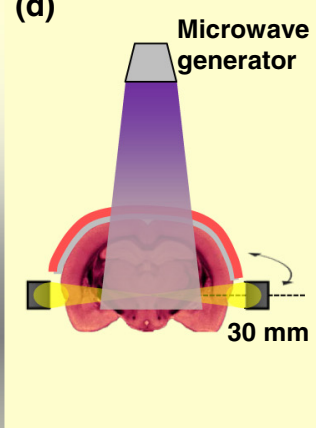

TAT (monkey)

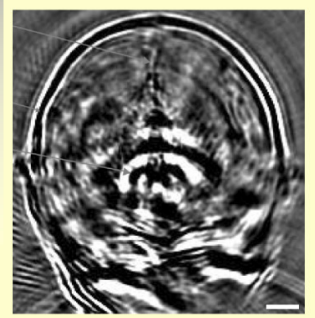

Fig. 1 Multiscale label-free photoacoustic (PA) brain imaging. (a) Optical-resolution photoacoustic microscopy (OR-PAM) of mouse cortical vasculature with the scalp removed but the skull intact. ${ }^{16}$ The optical lateral resolution of $\sim 3 \mu \mathrm{m}$ allows imaging the cortical blood vessel on the capillary level with a penetration depth of $<1 \mathrm{~mm}$. UT, ultrasonic transducer. (b) Acoustic-resolution PAM (ARPAM) of cortical vasculature in a living adult mouse with both the scalp and skull intact. ${ }^{17}$ A penetration depth of $\sim 3 \mathrm{~mm}$ can be achieved with an acoustic-diffraction-limited resolution of $\sim 70 \mu \mathrm{m}$. (c) Circularview photoacoustic computed tomography (PACT) of the cortical vasculature in a living adult rat with both the scalp and skull intact. ${ }^{18}$ With a $3.5 \mathrm{MHz}$ ultrasonic transducer, a penetration depth of $8 \mathrm{~mm}$ can be achieved, with an in-plane resolution of $0.2 \mathrm{~mm}$. (d) Circular-view thermoacoustic tomography (TAT) of a monkey brain with the scalp and skull intact. ${ }^{19}$ With microwave excitation, brain structures that are 3-cm deep in the head (e.g., the corpus callosum) can be clearly imaged with an in-plane resolution of $4 \mathrm{~mm}$. Sale bars: $1 \mathrm{~mm}$ for $(\mathrm{a}-\mathrm{c})$ and $1 \mathrm{~cm}$ for $(\mathrm{d})$. With laser excitation, hemoglobin in red blood cells provided the image contrast for (a)-(c). With microwave excitation, TAT imaged the water content in the brain in (d). Adapted with permission from Refs. 16, 17, 18, and 19. 
which is chosen to match the acoustic path length due to frequency-dependent acoustic attenuation. By adjusting the numerical aperture (NA) of the optical objective lens and/or the excitation wavelength, OR-PAM has achieved lateral resolutions ranging from $220 \mathrm{~nm}$ to $5 \mu \mathrm{m}$, with imaging depths ranging from $100 \mu \mathrm{m}$ to $1.2 \mathrm{~mm}$ in chicken breast tissue. ${ }^{11}$ Our experimental results show that the penetration depth of ORPAM at $570 \mathrm{~nm}$ in the mouse brain is $\sim 0.6 \mathrm{~mm}$ with skull intact and $1.0 \mathrm{~mm}$ without skull. The penetration depth can be improved by using near-infrared excitation at $1064 \mathrm{~nm}$.

Capitalizing on its high spatial resolution, OR-PAM can provide high-quality mouse cortical vasculature images using hemoglobin in red blood cells (RBCs) as the endogenous contrast. ${ }^{16}$ Due to the limited penetration depth of OR-PAM, the scalp of the mouse has to be removed, but the skull is kept intact. As shown in Fig. 1(a), cortical vasculature of a mouse brain is well resolved, down to capillaries. ${ }^{16}$ Therefore, OR-PAM is well suited for monitoring cortical hemodynamics as surrogates of the underlying neural activities.

OR-PAM is also suitable for single neuron imaging, using exogenous or endogenous contrast. For example, densely packed neuron cells cultured in medium were imaged by OR-PAM, assisted by immunochemistry staining of Tuj1 [Figs. 2(a) and 2(b)]. The single neuron cell body, dendrites, and nucleus can be clearly resolved. Alternatively, OR-PAM can image neurons by using lipids in the myelin as the endogenous imaging contrast. As shown in Fig. 2(c), the wavy fibrous structure in the OR-PAM image of an unsectioned sciatic nerve is caused by the bundles of myelin-coated axons that form the nerve fascicles. ${ }^{20}$ The bright round structures may be surrounding fat cells. Therefore, by using OR-PAM with appropriate contrast agents, it is possible to study single neuron activities, such as action potential propagation, neural transmitter release, and communications between synapses.

\subsection{Quasidiffusive PAT of the Brain}

Quasidiffusive PAT targets imaging depths in the quasidiffusive regime, which is more than one TMFP but less than 10 TMFPs. This imaging depth is ideal for small animal whole-brain imaging, since the entire mouse brain is about 5- to 7-mm thick. The core technologies involved in quasidiffusive PAT may not be fundamentally different from those used in ballistic or diffusive PAT, but modifications are needed to optimize the imaging performance for intermediate scale brain imaging. ${ }^{17}$

The representative quasidiffusive PAT implementation is acoustic-resolution photoacoustic microscopy (AR-PAM). ${ }^{21,22}$ With regard to the spatial resolution, AR-PAM can also be classified as a microscopic modality, because it can achieve a spatial resolution better than the resolving capability of the naked eye $(\sim 50 \mu \mathrm{m})$ when a high frequency ultrasonic transducer (e.g., $50 \mathrm{MHz}$ ) is used. ${ }^{21}$ Here, we consider AR-PAM as a "quasidiffusive" modality because it can break the optical diffusion limit.

Similar to OR-PAM, AR-PAM focuses the laser pulses to an area in tissue that coincides with the focal spot of a wideband ultrasonic detector [Fig. 1(b)]. However, unlike OR-PAM, the laser focus in AR-PAM is intentionally tuned wider than the ultrasonic focal spot, so that the entire volume of the ultrasonic focal zone is adequately illuminated. In this case, the resolution does not closely depend on the tissue's optical scattering characteristics, because it is not the optical focusing ability but the ultrasonic focusing that determines the resolution at depths within a few TMFPs. By adjusting the central frequency of the ultrasonic transducer and/or the NA of the acoustic focusing lens, the lateral resolution of AR-PAM can be scaled. As in ORPAM, the axial resolution of AR-PAM is determined by the bandwidth of the ultrasonic transducer.

Considering the acoustic properties of mouse scalp and skull, Stein et al. specially engineered AR-PAM for noninvasive imaging of the major mouse cortical vessels with both the scalp and skull intact [Fig. 1(b)]. By using a focused ultrasonic transducer with a central frequency of $20 \mathrm{MHz}$ and bandwidth of $90 \%$, AR-PAM has achieved a lateral resolution of $70 \mu \mathrm{m}$ and an axial resolution of $27 \mu \mathrm{m}$, with an imaging depth of more than $3.6 \mathrm{~mm}$ in the mouse brain. ${ }^{17}$

\subsection{Diffusive PAT of the Brain}

In the diffusive regime, photoacoustic computed tomography (PACT) is the major implementation for brain imaging. ${ }^{18}$ In contrast to OR-PAM/AR-PAM, where a focused single-element transducer is transversely scanned, PACT utilizes a state-ofthe-art transducer array to simultaneously detect the PA waves from the entire region of interest excited by an expanded laser beam [Fig. 1(c)]. Then, an inverse algorithm-essentially a method for sophisticated triangulation of PA sources from the detected time-resolved acoustic signals-is used to reconstruct
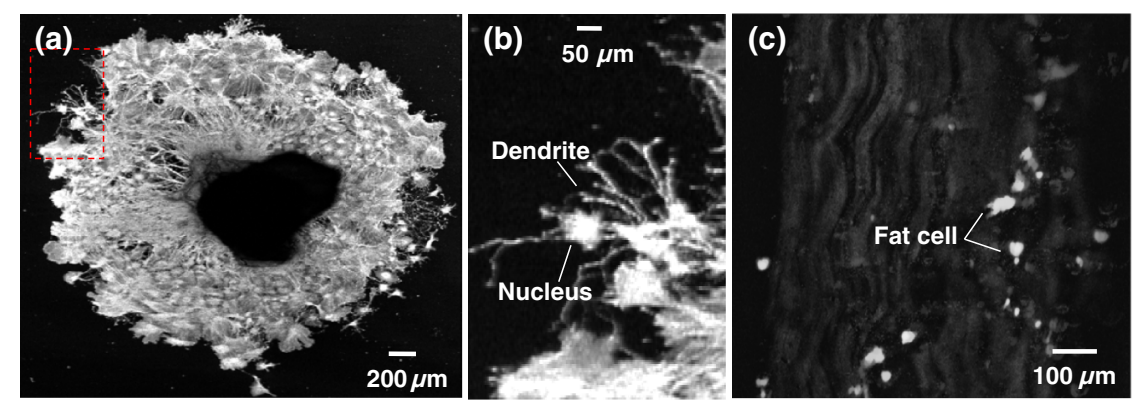

Fig. 2 PA microscopy of neurons. (a) OR-PAM of embryoid body-derived neurons, where neurofilaments were stained with anti-neurofilament/HRP-secondary antibody/DAB. (b) The close-up image of the dashed box region in (a) clearly shows the nucleus and detritus of a single neuron cell. (c) OR-PAM image of an unsectioned label-free sciatic nerve at $1210 \mathrm{~nm} .{ }^{20}$ The lipids in myelin provided the image contrast. The wavy fibrous structure in the image is caused by the bundles of myelin-coated axons that form the nerve fascicles. The bright round structures may be surrounding fat cells. Adapted with permission from Ref. 20. 
a high-resolution PACT image. Although a one-dimensional (1-D) array can provide one-dimensional (2-D) PA images, ${ }^{23}$ a 2-D array can perform three-dimensional PA imaging without extra mechanical scanning. ${ }^{24-26}$

Depending on the anatomy of the organ of interest, the transducer array in PACT can be constructed with different shapes, e.g., a circle ${ }^{23,27}$ or a plane ${ }^{25}$ for the brain, a hemisphere for the breast, ${ }^{28}$ and an arch for small animal trunks. ${ }^{29,30}$ As most ultrasonic arrays are 1-D, the axial and lateral resolutions of a PACT system are primarily determined by the bandwidth of the ultrasonic transducer and image reconstruction, ${ }^{31}$ whereas the elevational resolution usually comes from cylindrical acoustic focusing. ${ }^{9}$

PACT is ideal for deep brain imaging. The deep penetration of PACT is achieved by the combination of near-infrared (NIR) optical excitation and low frequency ultrasonic detection. A PACT system adapted from a clinical linear-array ultrasound machine operating at 4 to $8 \mathrm{MHz}$ has demonstrated an imaging depth of $7 \mathrm{~cm}$ in tissue phantom, with a lateral resolution of $720 \mu \mathrm{m}$ and an axial resolution of $400 \mu \mathrm{m}$. ${ }^{9}$ With the deep penetration, PACT has been extensively used for noninvasive brain imaging. In fact, the very first in vivo demonstration of PAT technology was to visualize the 3-D structures of a rat brain with both scalp and skull intact ${ }^{18}$ [Fig. 1(c)]. Interior brain structures $\sim 8 \mathrm{~mm}$ beneath the scalp surface were clearly imaged. ${ }^{18}$ In addition to small animal brains, PACT has successfully imaged rhesus monkey brains through a skull thickness of $\sim 2 \mathrm{~mm} .{ }^{32}$ A $1 \mathrm{MHz}$ (low frequency) ultrasonic transducer was used to receive PA waves that survived the acoustic attenuation and distortion by the relatively thick scalp and skull.

To further extend the imaging depth, radio frequency excitation can be used instead of laser excitation, a variant technology called thermoacoustic tomography [Fig. 1(d)]. Brain features $\sim 3-\mathrm{cm}$ deep in the monkey head were imaged with a resolution of $4 \mathrm{~mm} .{ }^{19}$ With this resolution and imaging depth, noninvasive human braining imaging is on the near horizon.

\subsection{Transitions Among Multiscale PAT Implementations}

Transitions among PAT implementations at different length scales merit a further discussion. As noted above, in PAT, the transition from ballistic through quasidiffusive to diffusive brain imaging is rather flexible and continuous, with significant overlap among various implementations. ${ }^{7}$

From a technology standpoint, PACT can be adapted for quasidiffusive imaging by using ultrasonic detectors with a higher central frequency and wider bandwidth. ${ }^{25,33}$ Likewise, AR-PAM can be adjusted for diffusive imaging by using low frequency ultrasonic detectors. For example, in AR-PAM, reducing the center frequency to $5 \mathrm{MHz}$ extends the imaging depth to $4 \mathrm{~cm}$ and relaxes the lateral resolution to $560 \mu \mathrm{m}$, an implementation called photoacoustic macroscopy (PAMac). ${ }^{34}$ Further, as optical focusing becomes gradually inefficient with increasing imaging depth, OR-PAM eventually transitions into AR-PAM. ${ }^{35}$

In addition, there are hybrid implementations. ${ }^{36,37}$ In a recently developed optical-resolution PACT system, the field of view is simultaneously excited by an array of 1800 diffraction-limited optical foci, and the resultant PA waves are detected by a 512-element transducer array. ${ }^{36}$ The parallel excitation and detection enable fast wide-field mouse brain imaging, while the imaging depth is restricted to one TMFP.
Overall, the flexible transitions among PAT implementations have provided a practical solution for multiscale brain imaging with the same imaging contrast.

\section{Functional PAT of the Brain in Action and at Rest}

Brain is the functional center of the human body, exerting centralized control over the other organs. Functional brain imaging is crucial for neuroscience studies, relating observable brain behaviors to underlying neural activities. Brain functions as a multilevel system, from neuron-to-neuron communications to column-to-column interconnections. PAT is promising for multilevel brain functional imaging. So far, PAT has been used in a number of brain studies and provided valuable information about brain oxygenation, brain metabolism, resting state brain connectivity, and brain responses to various physiological and pathological challenges. Here, we will present representative PAT brain studies and elaborate on the associated imaging technologies.

\subsection{PAT of Brain Oxygenation}

Oxygenation is an important indicator of the brain's functional status. When neuronal activity increases, there is an increased demand for oxygen, which triggers an increase in blood flow to the activated region. Generally, the blood flow increases not just to a level where oxygen demand is met, but to a level of overcompensation for the increased demand. Thus blood oxygenation actually increases following neural activation. The change in local blood oxygenation reflects the level of neural activity, which forms the principle of the blood oxygenation level dependent (BOLD) signals in fMRI. ${ }^{6}$ However, since the BOLD signal is sensitive only to the paramagnetic deoxy-hemoglobin (HbR), fMRI cannot distinguish between increased blood oxygenation and decreased blood perfusion (i.e., volumetric concentration of hemoglobin in the brain tissue). Therefore, to avoid this ambiguity, fMRI needs additional information about the change in volumetric concentration of hemoglobin in the brain tissue. In contrast, PAT is sensitive to both oxy-hemoglobin $\left(\mathrm{HbO}_{2}\right)$ and $\mathrm{HbR}$, and thus can measure blood oxygenation without ambiguity caused by the change in blood perfusion. ${ }^{38}$

From excitation fluence compensated PA measurements at two or more wavelengths, the relative concentrations of $\mathrm{HbO}_{2}$ and $\mathrm{HbR}$ can be quantified through spectral analysis, and thus the absolute oxygen saturation of hemoglobin $\left(\mathrm{sO}_{2}\right)$ can be computed. ${ }^{21}$ By using OR-PAM with two-wavelength measurements, $\mathrm{Hu}$ et al. quantified, for the first time, the mouse brain microvascular oxygenation down to capillaries, with the skull intact [Figs. 3(a) and 3(b)]. ${ }^{16}$ This study has opened a new window for neurovascular coupling research through the measurement of neuroactivity-dependent changes in hemoglobin concentration and oxygenation. Accurate excitation fluence compensation is less problematic for OR-PAM because of the superficial imaging depth. However, it is very challenging for AR-PAM and PACT in the quasidiffusive and diffusive regimes because of the wavelength-dependent light attenuation in tissue. ${ }^{27}$

To circumvent the fluence compensation issue in AR-PAM, Stein et al. reported a ratiometric method for relative oxygenation measurement [Figs. 3(c) and 3(d)]. ${ }^{39}$ In this method, the first measurement at $570 \mathrm{~nm}$ is insensitive to oxygenation, and the second measurement at $561 \mathrm{~nm}$ is $\mathrm{HbR}$ dominant. The pixelwise 


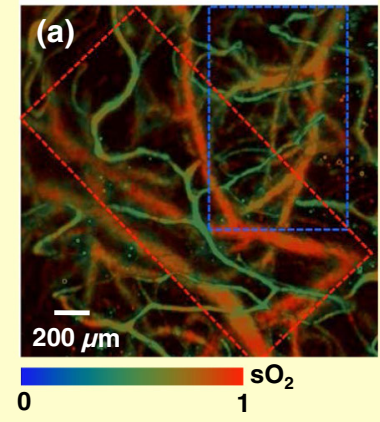

(b) $\mathrm{sO}_{2}(\%)$

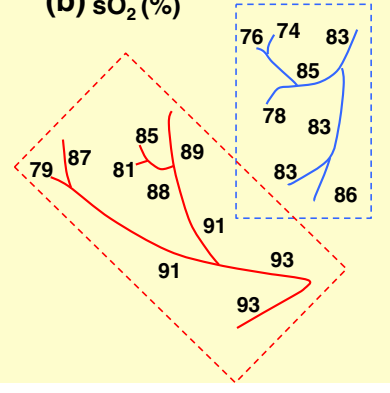

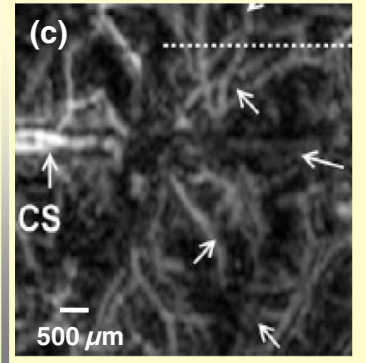
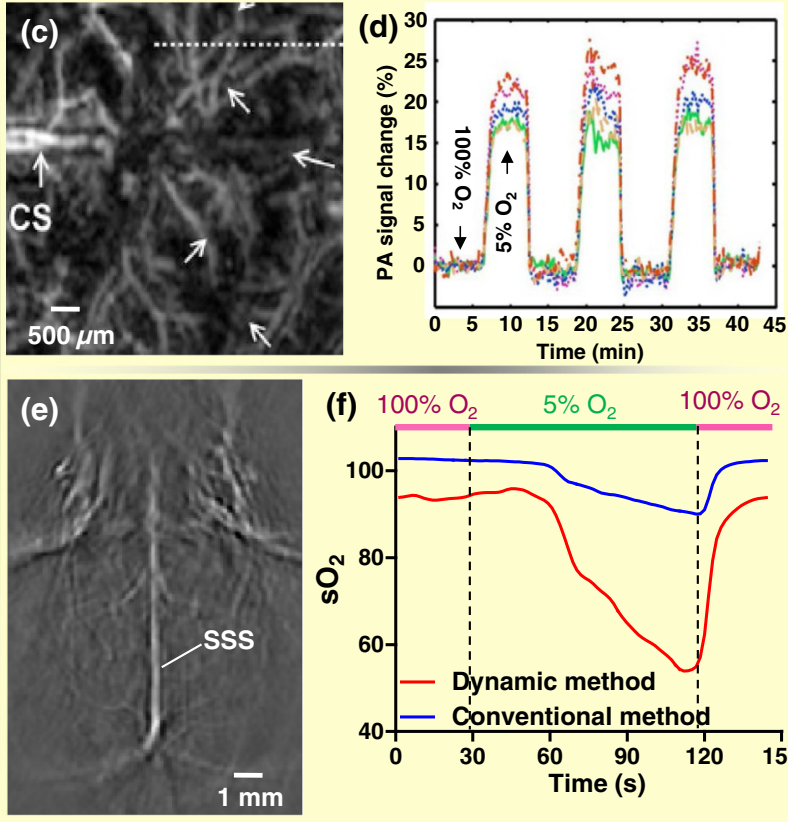

Fig. 3 Photoacoustic tomography (PAT) of mouse brain oxygenation. (a) OR-PAM of oxygen saturation $\left(\mathrm{sO}_{2}\right)$ in a mouse brain based on two-wavelength measurements at 570 and $578 \mathrm{~nm} .{ }^{16}(\mathrm{~b}) \mathrm{sO}_{2}$ values in percentage along an arterial tree and a venous tree marked by the dashed boxes in (a), showing decreased $\mathrm{sO}_{2}$ with vessel branch orders. (c) AR-PAM of cortical vasculature in a living mouse. ${ }^{39}$ The dotted white line indicates the line scanning range for oxygenation measurement. CS, coronal suture. (d) Dynamic vessel responses acquired through a hypoxic challenge, shown in percent change of ratiometric PA signals at 561 and $570 \mathrm{~nm}$. Each colored trace corresponds to the respective cortical vessel crossed by the dotted line in (c). (e) PACT of cortical vasculature in a living mouse. ${ }^{40}$ SSS, superior sagittal sinus. (f) Dynamics of absolute $\mathrm{sO}_{2}$ measured by PACT on the SSS in response to a hypoxic challenge. The measured $\mathrm{sO}_{2}$ values based on the new oxygenation-state method are compared with the conventional two-wavelength method. Adapted with permission from Refs. 16, 39, and 40.

ratio between the two measurements reflects the local blood oxygenation level, even though the local fluence is unknown. Although the resultant functional image is not a quantitative $\mathrm{sO}_{2}$ mapping, it is still useful when only the changes in blood oxygenation are of interest. With this method, ARPAM was used to monitor mouse cortical oxygenation transition between a hypoxic to a hyperoxic state. The dynamics of the transitions were clearly observed with a detection sensitivity of $3.6 \%$. Interestingly, the results show that the brain vasculature was much more resistant to transitioning from hyperoxia to hypoxia than the other way around. Using a similar method, Liao et al. studied mouse cortical oxygenation responses to electrical stimulations to the forepaws on a single vessel basis. ${ }^{41,42}$

Alternatively, without fluence compensation, the acoustic frequency spectra of PA signals at multiple optical wavelengths can be used to calculate absolute concentrations of $\mathrm{HbO}_{2}$ and $\mathrm{HbR}$, and thus $\mathrm{sO}_{2} \cdot{ }^{43,44}$ Recently, another calibration-free method for absolute $\mathrm{sO}_{2}$ quantification in PACT has been developed by Xia et al. based on the dynamics of the PA signals at different oxygenation states [Figs. 3(e) and 3(f)].$^{40}$ Briefly, at each oxygenation state, multiwavelength PA measurements are simultaneously performed, and the ratio of the PA measurements between different oxygenation states cancels out the effects of the heterogeneity of the laser fluence distribution as long as the local fluence does not change during the oxygenation transition. Therefore, absolute $\mathrm{sO}_{2}$ can be quantified without the need to correct for the local fluence. With this method, PACT was used to measure regional blood oxygenation in a mouse brain while the mouse was challenged from hyperoxia to hypoxia. Notably, the PACT measurement results also showed that the transition from hyperoxia to hypoxia took a longer time than the other way around, consistent with the observations from AR-PAM. ${ }^{39}$

\subsection{PAT of Brain Metabolism}

Brain metabolism is another important indicator of brain activities. In humans, the brain consumes $>20 \%$ of total energy, even in the resting state, and most of this energy is used to sustain neural activities. ${ }^{45}$ Since stronger neuron firing results in faster consumption of glucose and oxygen, tracking the metabolic rate of oxygen and glucose can provide valuable information about brain activities. Besides neural activities, abnormal brain metabolism may indicate diseases. In particular, hypermetabolism is a hallmark of all kinds of brain cancers, where the cancer cells are starving for oxygen and glucose. ${ }^{46}$ Since glucose can be metabolized via either aerobic respiration or anaerobic respiration, measurements of both glucose metabolism and oxygen metabolism are needed to fully reflect the metabolic status of brain tumors.

Positron emission tomography (PET) has been clinically used for brain metabolism measurement by injection or inhalation of radioactively labeled tracers. The detection sensitivity of PET is on the level of pM. However, the complex procedure, low resolution, high cost, and potential radiation exposure have limited its usage. On the other hand, PAT can measure the brain 
(a)

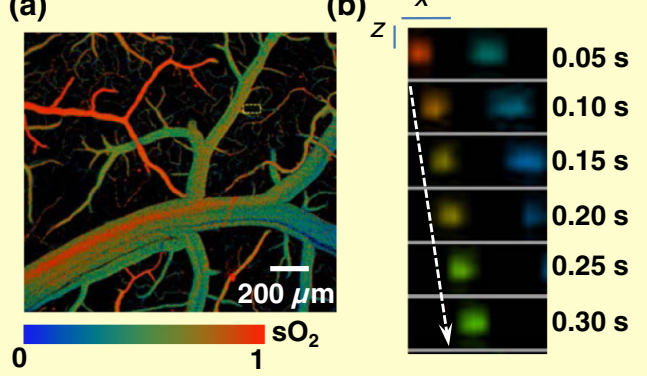

Fig. 4 Single cell label-free PA microscopy of oxygen metabolism in vivo. ${ }^{48}$ (a) $\mathrm{sO}_{2}$ mapping of the brain vasculature. The yellow dashed box indicates a capillary chosen for oxygen unloading measurement. (b) Single cell oxygen unloading was measured by fast line scanning along a capillary with two wavelength excitations. Blood flows from left to right. The dashed arrow follows the trajectory of a single flowing RBC. Scale bars: $x=10 \mu \mathrm{m}$ and $z=30 \mu \mathrm{m}$. Adapted with permission from Ref. 48.

metabolism of both oxygen and glucose with a better resolution, relatively low cost and less safety concern. ${ }^{47}$ Two examples follow.

By using hemoglobin as an endogenous oxygen tracer, ORPAM is capable of studying oxygen metabolism in the brain. Recently, by integrating fine spatial and temporal scales, single-cell PA flowoxigraphy, a new implementation of ORPAM, is capable of imaging oxygen release from single RBCs in vivo. ${ }^{48}$ As shown in Fig. 4, by fast line scanning $(20 \mathrm{~Hz})$ along a capillary with two wavelength excitations, PA flowoxigraphy can simultaneously measure multiple hemodynamic parameters that are required to quantify the oxygen release rate by RBCs, which is closely related to the local oxygen metabolism of neural cells. Experimental results show that PA flowoxigraphy can be used to image the coupling between neural activity and oxygen delivery in response to different physiological challenges, such as visual stimulation and acute systematic hypoglycemia. PA flowoxigraphy can be extremely useful in understanding how the brain is powered at the single cell level.

Although glucose has been explored as an endogenous contrast agent for PAT measurement of blood sugar levels, the detection sensitivity is still insufficient for clinical diagnosis, mainly due to strong background absorption by water. ${ }^{49}$ Recently, a glucose analog, 2-NBDG, has been used to noninvasively quantify glucose metabolism in a mouse brain. Similar to the FDG used in PET, 2-NBDG is transported into cells by glucose transporter GLUT. Inside cells, 2-NBDG is phosphatized, but cannot be further metabolized. Therefore, the distribution of trapped 2-NBDG reflects the glucose uptake and thus the local glucose metabolism. PACT with 2-NBDG was used for noninvasive mouse brain metabolism studies, ${ }^{50}$ where the glucose uptake rate in the somatosensory region was elevated by $\sim 3 \%$ in response to electrical stimulations to the mouse hindpaws. 2-NBDG is suited for brain imaging because it can freely pass blood-brain barrier (BBB) due to its relatively small molecule size. However, 2-NBDG has peak absorption at $480 \mathrm{~nm}$. Because of strong light attenuation by brain tissue at $480 \mathrm{~nm}$, 2-NBDG is not ideal for deep brain imaging. In addition, it is still under investigation whether most of the existing optical glucose analogs share the same transport mechanism into the brain as glucose. ${ }^{51}$ Alternatively, newly developed FRET-based glucose sensors, especially genetically encoded fluorescent proteins, can be expressed in the brain cells and do not need transport across BBB, and thus can be adopted for PA imaging of glucose metabolism in the future. ${ }^{52,53}$

\subsection{PAT of Brain Resting-State Connectivity}

The mammalian brain is subdivided into specialized regions for various functions. These functional regions show pronounced activities even in the resting state. The spatiotemporal correlations between the spontaneous activities in different regions stem from the anatomical connections and the underlying neural activities in the brain cortex..$^{54,55}$ fMRI and diffuse optical tomography (DOT), the most commonly used functional brain imaging tools for resting state studies, lack the spatial and/or temporal resolution for small animal brain imaging.

Using the full-ring-array PACT system, Nasiriavanaki et al. successfully imaged functional connectivity imaging in the resting mouse brain, with an in-plane resolution of $100 \mu \mathrm{m}$ and a frame rate of $0.6 \mathrm{~Hz}$ (Fig. 5). ${ }^{56}$ The results clearly indicate bilateral correlations between the main functional regions

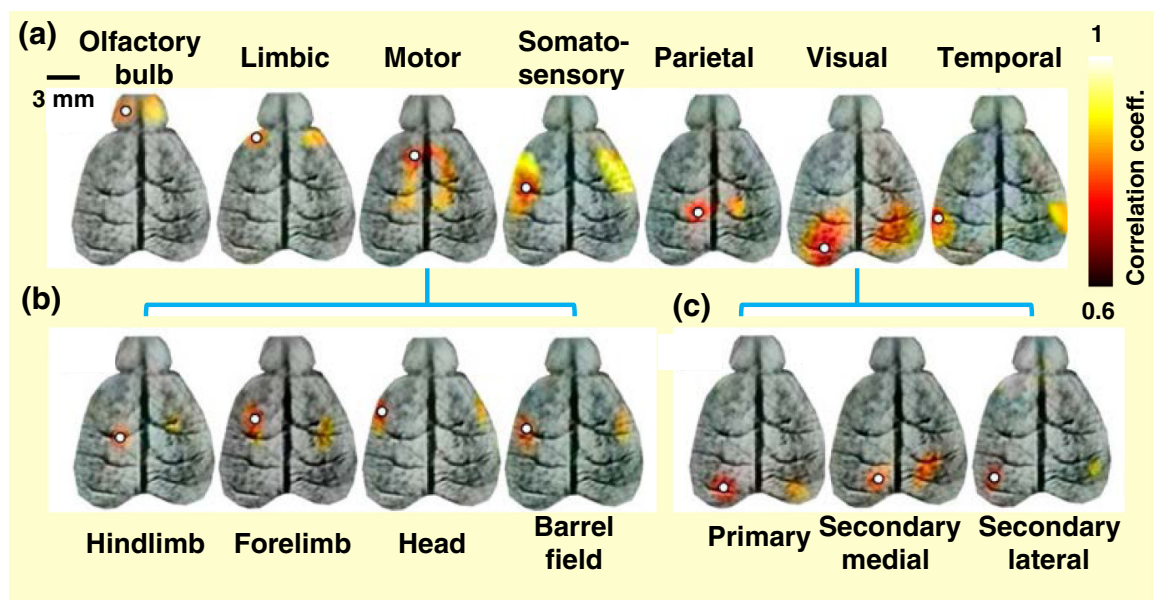

Fig. 5 Functional resting-state connectivity maps in a live mouse brain acquired noninvasively by PACT. ${ }^{56}$ Correlation maps of (a) the main functional regions, (b) the four subregions of the somatosensory cortex, and (c) the three subregions of the visual cortex. The white circles in the images are the seed locations for the correlation calculation. Adapted with permission from Ref. 56. 
[Fig. 5(a)], as well as in several subregions [Figs. 5(b) and 5(c)]. The functional connectivity maps by PACT are automatically coregistered with high-resolution cortical vascular images, allowing pinpoint location of neural activities. Although not demonstrated yet, by using spectral analysis, PACT can potentially unmix the contributions of $\mathrm{HbO}_{2}$ and $\mathrm{HbR}$ to the functional connectivity, which cannot be achieved by fMRI.

\subsection{PAT of Brain Responses to Physiological Challenges}

In addition to resting state brain studies, PAT has also been extensively used to image brain responses to various physiological challenges in small animals, which may provide valuable insights into the human brain. PAT can provide morphological, functional, and metabolic imaging of the brain at different spatiotemporal scales.

For example, brain responses to oxygenation challenges have been imaged by PAT across the scales. Although the scalp was removed for OR-PAM, both the scalp and skull were intact for AR-PAM and PACT. Capitalizing on high spatial resolution, OR-PAM is able to scrutinize the oxygenation transitions in single capillaries or even in single RBCs; however, the imaging depth is limited to $<1 \mathrm{~mm} .{ }^{48}$ AR-PAM can image brain oxygenation transitions at depths up to a few millimeters, but only cross-sectional images can be repeatedly acquired, limited by its temporal resolution. ${ }^{39}$ In contrast, full-ring PACT can image the oxygenation transitions over the entire mouse brain cortex at a high imaging speed, but with compromised spatial resolution. ${ }^{40}$

Small animal brain responses to other physiological challenges have also been imaged by PAT, including applying electrical stimulations to paws [Fig. 6(a)], ${ }^{41,50}$ vibrational stimulations to whiskers [Fig. 6(b)], ${ }^{18}$ optical stimulations to eyes [Fig. 6(c)], ${ }^{48}$ direct electrical stimulations to brains, ${ }^{57}$ and injection of cocaine hydrochloride. ${ }^{58}$

\subsection{PAT of Small Animal Models for Brain Disorders}

Over the years, many small animal models have been developed for studying human brain diseases, including brain tumors, stroke, epilepsy, traumatic brain injury, Parkinson's disease, Huntington's disease, and Alzheimer's disease. ${ }^{3}$ By combining appropriate imaging techniques with suitable small animal models, it has become possible to investigate the underlying mechanisms of brain diseases in a controlled manner and on a much finer scale than is possible in humans. ${ }^{59,60}$

So far, PAT has been applied in studies of a number of brain disease models. Li et al. were the first to apply functional PACT to image a human glioblastoma at $\sim 3$-mm depth in mouse brains [Fig. 7(a)]. ${ }^{61}$ The increased total hemoglobin concentration in the tumor region indicated tumor angiogenesis, and the decreased oxygen saturation suggested a hypoxic tumor vasculature: both are hallmarks of late-stage cancers. Staley et al. were the first to demonstrate noninvasive longitudinal monitoring of melanoma growth and metastasis in a mouse brain by using PAMac. ${ }^{62}$ The strong absorption of melanin provided an excellent signal-to-noise ratio of $\sim 30 \mathrm{~dB}$, with the tumor implanted $\sim 3 \mathrm{~mm}$ beneath the skull.

$\mathrm{Xu}$ et al. were the first to apply PACT to study mouse cerebral edema induced by cold injury [Fig. 7(b)]. In this study, the formation, expansion, and recovery of edema were
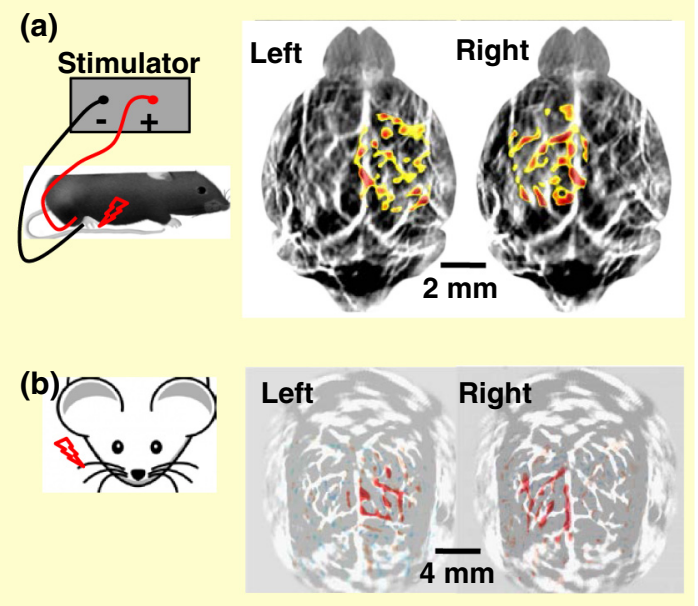

(c)
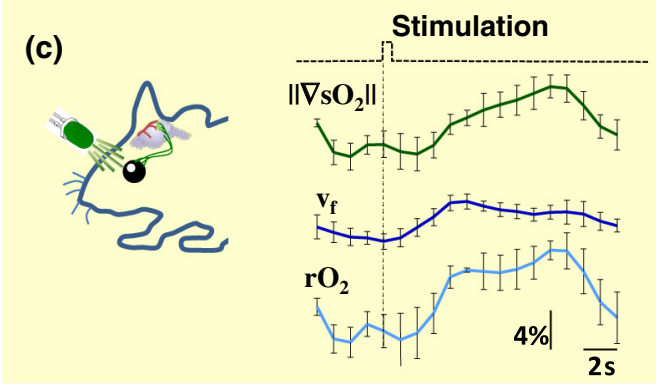

Fig. 6 PAT of brain hemodynamics in responses to stimulations. (a) PACT of mouse brain hemodynamics in response to electrical stimulations on the hindpaws showing the elevated blood perfusion in the contralateral somatosensory regions. ${ }^{50}$ The PA signal increases (in color) are superimposed on the vascular image (in gray). (b) PACT of rat brain hemodynamics in response to vibrational stimulations on the whiskers, showing the increased blood perfusion in the contralateral somatosensory region. ${ }^{18}$ The PA signal increases (in red color) are superimposed on the vascular image (in gray). (c) OR-PAM of neuron-RBC coupling in the mouse visual cortex. ${ }^{48}$ The eye of a mouse was stimulated by a flashing LED (left), and transient responses to a single visual stimulation were monitored. Clear increases were observed in the magnitude of the $\mathrm{sO}_{2}$ gradient $\left(\| \nabla \mathrm{sO}_{2}||\right)$, blood flow speed $\left(\mathrm{v}_{\mathrm{f}}\right)$ and oxygen unloading rate $\left(\mathrm{rO}_{2}\right)$. Adapted with permission from Refs. 18, 48, and 50.

noninvasively monitored by PACT, where water content in the edema provided PA image contrast at $975 \mathrm{~nm}^{63}$ Significantly, this study demonstrated the potential of in vivo water imaging by PAT. Hu et al. were the first to apply ORPAM to longitudinally study ischemic stroke induced brain injury in a mouse model [Fig. 7(c)]. ${ }^{64}$ The focal brain ischemia was caused by 1-h transient middle cerebral artery occlusion (MCAO). A significant drop in $\mathrm{sO}_{2}$ was observed in the core stroke region right after $\mathrm{MCAO}$, which eventually led to brain infarction after 24 days.

Zhang et al. and Tsytsarev et al. explored noninvasive or minimally invasive imaging of drug-induced epileptic seizures in animal models using PACT (Ref. 65) and OR-PAM (Ref. 66), respectively. With PACT's wide field of view, it was found that the seizures induced an increase in the PA signal confined in the epileptic core region. However, limited by the spatial resolution of PACT, it was unknown whether the increase in PA signal was caused by an increase in vessel diameters, total hemoglobin concentration, or both. This question was later resolved in a study with OR-PAM, where an artery-vein pair 
(a)

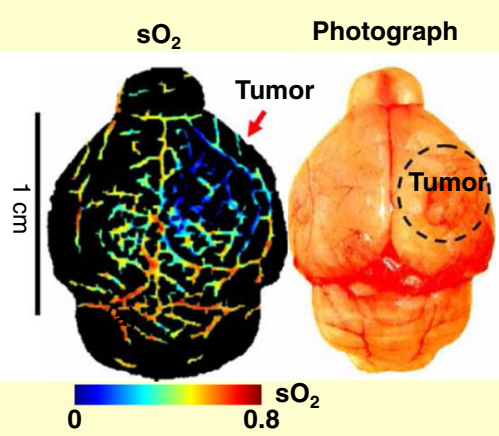

(b)

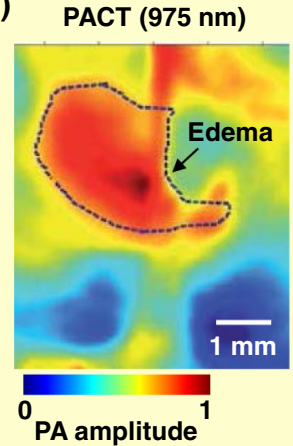

MRI (T2)

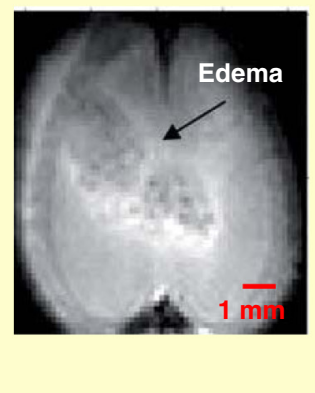

(c)
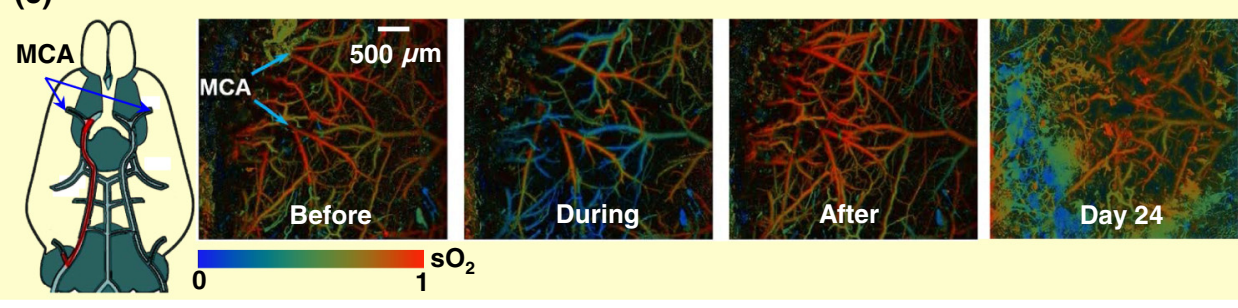

Fig. 7 Noninvasive PAT of small animal disease models. (a) Functional PACT of a glioblastoma in a mouse brain showing the hypoxic tumor region (left). ${ }^{61}$ The tumor region was confirmed by a postmortem photograph of the excised mouse brain (right). (b) PACT of cold-injury induced edema in a mouse brain, where the water content provided the image contrast at $975 \mathrm{~nm}$ (left).$^{63}$ The edema region was confirmed by the MRI T2 image. (c) Longitudinal transcranial OR-PAM monitoring of cerebral $\mathrm{sO}_{2}$ during strokeinduced brain damage. ${ }^{64}$ The stroke was induced by 1 -h transient middle cerebral artery occlusion (MCAO). $\mathrm{sO}_{2}$ images were acquired before, during, right after, and 24 days after the MCAO. MCA, middle cerebral artery. Adapted with permission from Refs. 61, 63, and 64.

was imaged. ${ }^{66}$ The seizures induced simultaneous increases in the vessel diameters and total hemoglobin concentrations in both the artery and the vein. Compared with the vein, the increase in the artery had a reduced amplitude but a prolonged time. The cross-validation of the epileptic observations at different length scales is a good example of the powerful multiscale imaging capability of PAT in brain research.

Another disease model, norepinephrine induced brain hypertension, was studied by Liu et al. with OR-PAM ${ }^{67}$ By virtue of the high spatiotemporal resolution and multiparametric imaging capability of OR-PAM, for the first time, the effects of norepinephrine on the brain hemodynamics were investigated on a single vessel level. The key findings showed clear causal consequences following the drug administration: severe vessel constriction resulted in reduced blood perfusion, which was followed by a drop in oxygen saturation.

Many other small animal models have been used for PA brain imaging. ${ }^{68-70}$ Collectively, these small animal disease models have demonstrated the great potential of PAT for monitoring the brain's physiological and pathological status, and in assessing the effects of treatments.

\section{PA Molecular Imaging in the Brain}

In mammals, the brain is largely composed of water (w/w 78\%), lipids $(12 \%)$, proteins $(8 \%)$, and other substances $(2 \%) .^{71}$ Compared with other internal organs, the brain has a relatively high content of lipids. Particularly, the dry mass of myelin is $70 \%$ to $85 \%$ lipids. Hemoglobin is about $1.5 \%$ of the total brain mass. ${ }^{71}$ All these endogenous components can be imaged by PAT at their absorption wavelengths. So far, hemoglobin is most widely used in PA hemodynamic imaging of brain. ${ }^{8}$ As discussed above, water has also been explored in PAT of brain edema. ${ }^{72}$ Lipids in myelin have been imaged in peripheral nerves ${ }^{20}$ and can be potentially used for imaging the central nervous system.

In addition to these endogenous contrasts, PAT can utilize exogenous contrasts for molecular brain imaging with enhanced specificity and sensitivity. Molecular imaging enables visualizing the cellular function and the molecular processes. PA molecular imaging differs from traditional PA imaging, in that targeting molecules (e.g., antibodies) are conjugated to the imaging probes and used to help image particular targets or pathways. In recent years, great efforts have been devoted to promote the molecular imaging capability of PAT, and considerable progress has been made in optimizing both the PAT imaging systems for better detection sensitivity, ${ }^{73-75}$ signal processing methods for better spectral unmixing, ${ }^{43,44,76}$ and the contrast agents for better contrast enhancement. ${ }^{77-80}$ Notably, a new technology referred to as multispectral optoacoustic tomography has been actively used for PA molecular imaging. ${ }^{80,81}$ PAT has proven capable of high sensitivity molecular imaging by using various exogenous contrast agents, including nanoparticles, organic dyes, fluorescent proteins, and reporter gene products. 82

However, unlike other organs, the brain presents a significant obstacle for molecular imaging: the BBB..$^{83,84}$ The BBB protects the brain from noxious agents; however, it also significantly hinders the delivery of contrast agents to the brain. Through the tight junctions between capillary endothelial cells, the BBB blocks the diffusion of nearly $100 \%$ of microscopic objects (e.g., bacteria) and $>95 \%$ of large or hydrophilic molecules into the cerebrospinal fluid (CSF). ${ }^{85}$ For ideal brain molecular imaging, the exogenous contrast agents need to cross the BBB in adequate quantity and remain in the brain for a sufficient time. So far, various methods have been used to break, bypass or 
"trick" the BBB in order to deliver contrast agents into the brain. ${ }^{83}$ These include physically or chemically opening the $\mathrm{BBB}$, direct delivery into the $\mathrm{CSF}$, and active transport by intrinsic carriers.

Due to the relatively small molecule size $(<10 \mathrm{~nm})$, organic dyes may be delivered through the BBB by active transport. As discussed, 2-NBDG, a glucose analog, can be transported across the BBB via glucose transport protein GLUT [Fig. 8(a)]. ${ }^{86,87}$ The active transport is safe for the barrier, and the passage is well regulated by the available transport carriers. The disadvantage is that only a small number of organic dyes can be transported ${ }^{88}$ For example, another glucose analog, IRDye800-DG, may not be actively transported due to its large molecule size. ${ }^{89}$

In addition to active transport, the BBB can also be chemically or physically opened to allow the free diffusion of contrast agents. ${ }^{91} \mathrm{Li}$ et al. used IRDye800-c(KRGDf) to target the overexpressed integrin $\alpha_{\mathrm{v}} \beta_{3}$ in brain glioblastoma. ${ }^{61}$ Before the administration of IRDye800-c(KRGDf), the mouse brain BBB was opened by intravenous injection of mannitol, which could temporarily shrink the endothelial cells and simultaneously stretch the tight junctions between them [Fig. 8(b)]. ${ }^{92}$ PAT achieved a detection sensitivity of $\sim 250 \mathrm{nM}$ of IRDye800-c (KRGDf), with an incident laser fluence of $20 \mathrm{~mJ} / \mathrm{cm}^{2}$ at
$784 \mathrm{~nm}$. However, the drawbacks of forcibly opening the $\mathrm{BBB}$ include potential damage to the barrier as well as uncontrolled passage of contrast agents into the brain.

Compared with organic dyes, nanoparticles are relatively large $(>10 \mathrm{~nm})$ and thus more difficult to transport across the BBB. ${ }^{84}$ However, nanoparticles can be more easily engineered for PA molecular imaging; in particular, the peak absorption wavelength can be easily tuned. ${ }^{78,79,93,94}$ Numerous nanoparticles with various sizes, shapes, and compositions have been used for PA molecular imaging, especially in vascular enhancement, ${ }^{95,96}$ early cancer detection, ${ }^{97-99}$ drug delivery, ${ }^{100,101}$ and sentinel lymph node mapping. ${ }^{102,103}$ However, only a few of them have been explored for PAT of the brain, mostly due to the difficulty of passing the BBB.

Nevertheless, in recent years, different methods have been studied for controlled nanoparticles delivery to the brain. ${ }^{83}$ Kircher et al. developed a brain tumor molecular imaging method using triple-modality MRI-photoacoustic-Raman nanoparticles. ${ }^{90}$ In this method, gold-silica-based nanoparticles coated with $\mathrm{Gd}^{3+}$ ions enhanced PA imaging of glioblastoma in a mouse brain with an intact scalp and skull. Here, the nanoparticles diffused through the disrupted BBB and accumulated in tumor cells without the need for a specific targeting
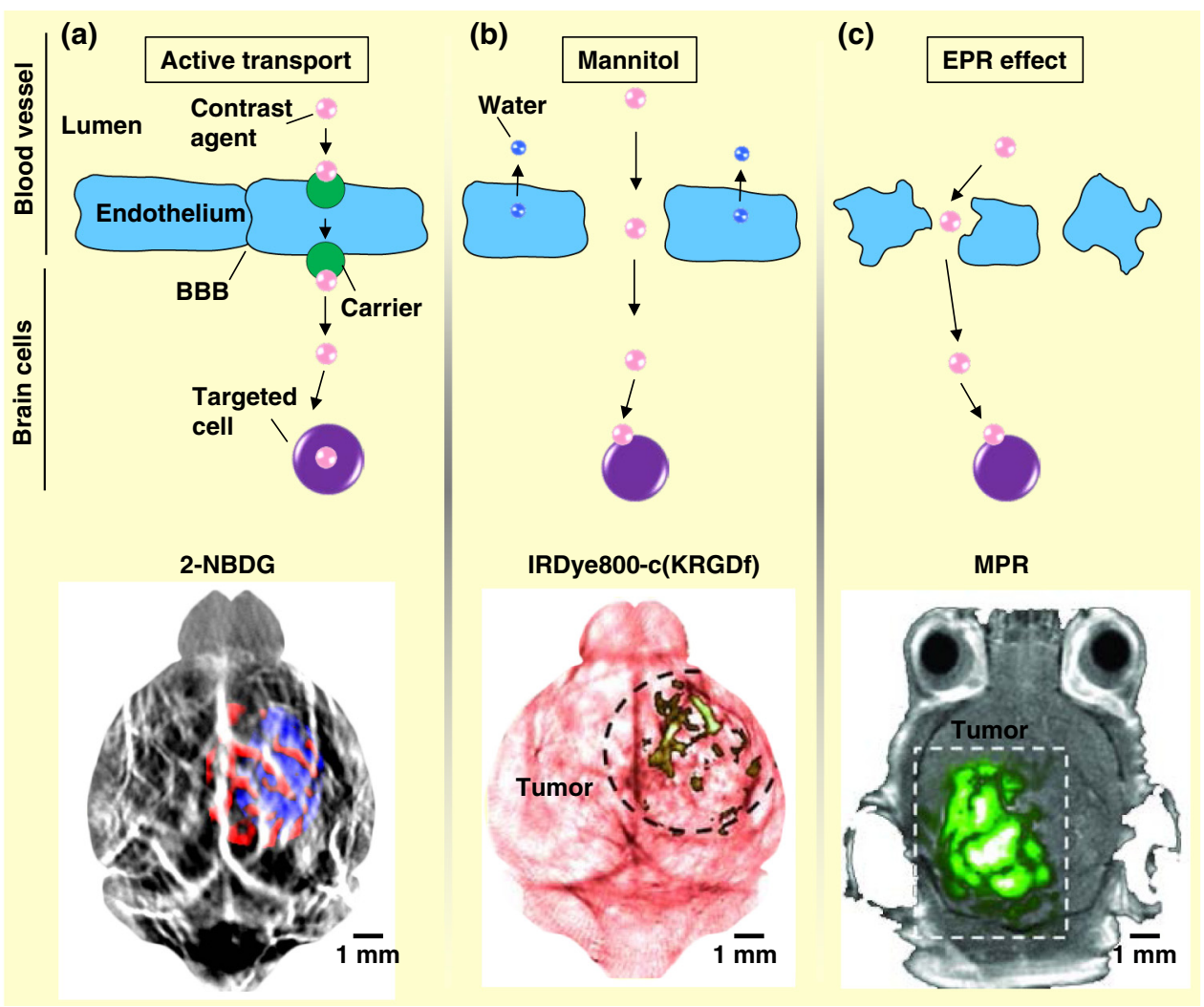

Fig. 8 PA molecular imaging of the mouse brain. (a) PACT of glucose metabolism in a mouse brain, by using 2-NBDG as the contrast agent..$^{50}$ 2-NBDG was delivered across the blood-brain barrier (BBB) via active transport by glucose transport protein GLUT. (b) PACT of a glioblastoma in a mouse brain enhanced by IRDye800-c(KRGDf), which targeted the overexpressed integrin $\alpha_{v} \beta_{3}$ in tumor cells. ${ }^{18}$ The BBB was forcibly opened by intravenous injection of mannitol, which temporarily shrank the endothelial cells and simultaneously stretched the tight junctions of the BBB. (c) PAMac of a glioblastoma in a mouse brain enhanced by trimodality MRI-photoacoustic-Raman nanoparticles. ${ }^{90}$ Here, the nanoparticles diffused through the disrupted BBB and accumulated in tumor cells without the need for a specific targeting mechanism, a phenomenon referred to as the enhanced permeability and retention (EPR) effect. Adapted with permission from Refs. 50, 61, and 90. 
mechanism, a phenomenon referred to as the enhanced permeability and retention (EPR) effect [Fig. 8(c)]. ${ }^{90,104}$ A PA detection sensitivity of $50 \mathrm{pM}$ was achieved with an incident laser energy of $8 \mathrm{~mJ} / \mathrm{cm}^{2}$. However, the EPR effect is considered a widely variable phenomenon that highly depends on the local tumor environment and cannot be relied on for robust delivery of nanoparticles across the BBB. ${ }^{83}$

Compared with organic dyes and nanoparticles, reporter gene products have a significant advantage for brain imaging: the gene products are expressed in living brain cells and do not need complex exogenous delivery across the BBB. So far, various fluorescent genetically encoded proteins have been explored for PA molecular imaging, such as mCherry, EGFP, iRFP, and RFP. ${ }^{105-109} \mathrm{Li}$ et al. reported the first PA reporter gene imaging in rat brains, where gliosarcoma cells expressing LacZ gene were imaged by PACT using the blue product as the contrast agent. ${ }^{110}$ The blue product was produced from LacZ encoded $\beta$-galactosidase and locally injected X-gal. A detection sensitivity of $500 \mathrm{nM}$ was demonstrated with an incident laser fluence of $5 \mathrm{~mJ} / \mathrm{cm}^{2}$. A drawback of LacZ gene imaging is that $\mathrm{X}$-gal needs to be injected locally, which may cause brain damage. Recently, using multispectral PAT, Razansky et al. demonstrated PAT of mCherry in the brain of an adult (6-month-old) transgenic zebrafish, where no extra imaging substances were needed. ${ }^{105}$

However, compared with organic dyes, most fluorescent proteins have relatively low extinction coefficients and low photostability, and thus are not ideally suited as PA genetic reporters. ${ }^{106}$ To resolve this issue, given the strong optical absorption of melanin, a few groups have been working on transferring tyrosinase genes to nonmelanogenic cells to encode eumelanin as the contrast agent for PA imaging. However, the melanin synthesis usually consumes considerable cellular resources and may jeopardize normal cell functions. ${ }^{11-114}$

\section{Challenges for PAT of the Human Brain}

Clinical translation of PAT to human brain imaging is one of its most exciting directions. With better resolution than that of fMRI and richer functional information than that of ultrasound, PAT is expected to become a powerful tool for understanding various neural activities and cognitive phenomena. However, despite the successes in small animal brain imaging, PAT of the human brain still faces challenges. The major obstacle is that the incident light and the emitted ultrasound need to go through the thick human skull ( $\sim 7$ to $11 \mathrm{~mm}$ for adolescents and adults), which is much thicker than a mouse skull $(\sim 0.2$ to $0.3 \mathrm{~mm})$, a rat skull $(\sim 0.7$ to $1 \mathrm{~mm})$, or a monkey skull ( $\sim 2$ to $4 \mathrm{~mm})$. The skull can strongly absorb and scatter light, and attenuate and distort ultrasound as well.

The strong optical attenuation of the skull is largely due to its high optical scattering. ${ }^{115}$ The reduced scattering coefficient of human skull in NIR region is $\sim 1.3$ to $2.7 \mathrm{~mm}^{-1}$. The direct light transmittance at $1064 \mathrm{~nm}$ through the top of the fixed human skull is only $\sim 2.1 \%{ }^{116}$ Since the American National Standards Institute safety standard requires the incident light fluence at the skull surface to be $<100 \mathrm{~mJ} / \mathrm{cm}^{2}$ in the NIR region, the maximum delivered fluence at the brain surface is about $2 \mathrm{~mJ} / \mathrm{cm}^{2}$.

The detection phase suffers strong acoustic attenuation of the skull $(\sim 20 \mathrm{~dB} / \mathrm{cm}$ at $1 \mathrm{MHz})$, which is about 25 times that of brain tissue $(0.8 \mathrm{~dB} / \mathrm{cm}) .{ }^{17,118}$ In addition to degrading the SNR, the skull also introduces severe acoustic signal aberration associated with acoustic wave reflection and refraction within the skull. ${ }^{119}$ The acoustic aberration is due to the acoustic impedance mismatch between the skull $\left(\sim 7.7 \times 10^{6}\right.$ Rayls $)$ and the brain $\left(\sim 1.6 \times 10^{6}\right.$ Rayls $),{ }^{117}$ and the distorted acoustic waves can significantly deteriorate the reconstructed image quality.

Although the above challenges have hindered the progress in PAT of the human brain, concerted efforts have been made to improve the image quality. To optimize the incident light delivery, Nie et al. have developed a novel photon cycler for PACT to recycle photons back-scatted by the skull. The recycler is coated with titanium white pigment and can effectively reflect the photons back to the brain to generate PA signals. ${ }^{116}$ An ex vivo experiment with a canine brain covered by a human skull showed that the photon recycler improved the SNR by 2.4 times.

Furthermore, due to the relatively low optical absorption of the skull in the NIR region, NIR contrast agents can be used to improve the SNR. Wang et al. used polyethylene glycol conjugated indocyanine green (ICG), which absorbs strongly at $790 \mathrm{~nm}$, to enhance hemodynamic imaging in the rat brain with intact scalp and skull. ${ }^{120}$ A detection sensitivity of $10 \mu \mathrm{M}$ was demonstrated with an incident laser fluence of $2 \mathrm{~mJ} / \mathrm{cm}^{2}$. Since ICG is an FDA approved contrast agent for determining human cardiac output, ${ }^{121}$ it may also be used for human brain imaging.

Because the high frequency signal is attenuated faster than the low frequency signal, much of the PA signal emitted through a human skull resides at frequencies lower than $1 \mathrm{MHz}$. Therefore, an ultrasonic detection system that matches the PA signal bandwidth can further improve the SNR. ${ }^{118,119}$

In addition to the engineering improvements to PAT systems, image reconstruction methods have also been improved to correct for the acoustic signal distortions. Huang et al. have used adjunct X-ray CT data of a skull to extract speed of sound (SOS) and mass density, from which the acoustic impedance of the skull can be calculated. ${ }^{119}$ Their phantom experiments showed that, by incorporating these quantities into the time-reversal image reconstruction, skull-introduced signal aberrations were corrected, and thus the reconstructed image artifacts were reduced. Similarly, Xia et al. have incorporated ultrasonic computed tomography with PACT to correct for SOS heterogeneity within the field of view, which can substantially improve the image quality especially for large targets. ${ }^{122}$

\section{Conclusions and Outlook}

Brain mapping will not just unlock the mystery of how the brain works, but also create a platform for better diagnostics and therapeutics of brain disorders. ${ }^{3}$ Although many technologies have been used for brain imaging, there is still a large role for PAT to play, complementing other modalities. Particularly, the high scalability of PAT will enable high resolution brain imaging at length scales ranging from single neurons to individual functional regions, using the same optical absorption contrast. The functional imaging capability of PAT will help to address how the brain operates in normal conditions and why it falls into disorders.

As a maturing technology, PAT has not yet realized its utmost potentials in brain imaging. For small animal brain imaging, PAT of awake animals will open a new door to many investigations that can bridge the gap between neural activity and behavior. Further miniaturizing PAT systems with microfabrication technologies may allow mounting the imaging system on the 
animal's head without jeopardizing the animal's normal activity. ${ }^{123,124}$ Alternatively, head restraint may be possible using a standard PAT system. For example, an air-supported spherical treadmill may be adapted for PAT of awake mice. ${ }^{125}$ Moreover, direct neural activities in vivo can be photoacoustically detected by using voltage-sensitive dyes or calcium-sensitive dyes, whose fluorescence emission changes when a neuron fires an action potential. ${ }^{105,126}$

Human brain imaging will remain as one of the most important tasks of PAT, and is expecting breakthroughs in the coming years. Novel light delivery methods are needed to improve the illumination efficiency. To bypass skull attenuation, the nose, ear, and mouth cavities may be used to deliver light to the deep brain. In contrast to adult brain, neonatal brain PAT should be much less challenging due to the thinner skull and the soft fontanel. Novel reconstruction methods assisted by X-ray CT or MRI can correct for the acoustic signal distortions by the skull. More PAT contrast agents with strong optical absorption and high biocompatibility will be developed to specifically target the brain. New methods for delivering the contrast agents through the $\mathrm{BBB}$ will be explored, for example, by direct delivery through the nasal cavity. Eventually, building on advances in imaging systems, reconstruction methods and contrast agents, functional human brain imaging by PAT will become highly possible.

In conclusion, considering its highly scalable spatial resolution, imaging speed, penetration depth, and functionality, we expect that PAT will become a promising tool for fundamental neurophysiological studies and clinical neurological practice.

\section{Acknowledgments}

The authors appreciate professor James Ballard's close reading of the paper, and Yu Zhang and professor Younan Xia (Georgia Institute of Technology) for providing the cultured neuron sample in Figs. 2(a) and 2(b). This work was sponsored by NIH Grants DP1 EB016986 (NIH Director's Pioneer Award), R01 CA186567 (NIH Director's Transformative Research Award), R01 EB016963, R01 CA134539, R01 EB010049, R01 CA157277, and R01 CA159959. L.V. Wang has a financial interest in Endra, Inc., and Microphotoacoustics, Inc., which, however, did not support this work.

\section{References}

1. M. E. Raichle, "Functional brain imaging and human brain function," J. Neurosci. 23(10), 3959-3962 (2003).

2. M. E. Raichle, "A paradigm shift in functional brain imaging," J. Neurosci. 29(41), 12729-12734 (2009).

3. T. R. Insel, S. C. Landis, and F. S. Collins, "Research priorities. The NIH brain initiative," Science 340(6133), 687-688 (2013).

4. A. Devor et al., "The challenge of connecting the dots in the BRAIN," Neuron 80(2), 270-274 (2013).

5. F. Helmchen and W. Denk, "Deep tissue two-photon microscopy," Nat. Methods 2(12), 932-940 (2005).

6. S. Ogawa et al., "Brain magnetic-resonance-imaging with contrast dependent on blood oxygenation," Proc. Natl. Acad. Sci. U. S. A. 87(24), 9868-9872 (1990).

7. V. Ntziachristos, "Going deeper than microscopy: the optical imaging frontier in biology," Nat. Methods 7(8), 603-614 (2010).

8. S. Hu and L. V. Wang, "Neurovascular photoacoustic tomography," Front. Neuroenerg. 2, 10 (2010).

9. L. H. V. Wang and S. Hu, "Photoacoustic tomography: in vivo imaging from organelles to organs," Science 335(6075), 1458-1462 (2012).

10. M. Soleimani and R. H. Bayford, "New and emerging tomographic imaging techniques in medical and industrial applications (vol 367, pg 3017, 2009)," Philos. Trans. R. Soc. A 367(1904), 4137-4137 (2009).

11. J. Yao and L. V. Wang, "Photoacoustic microscopy," Laser Photonics Rev. 7(5), 758-778 (2013).

12. L. V. Wang, "Multiscale photoacoustic microscopy and computed tomography," Nat. Photonics 3(9), 503-509 (2009).

13. P. H. Raven and G. B. Johnson, Biology, 3rd ed., p. 82, Wm. C. Brown Publishers, Dubuque, Iowa (1995).

14. K. Maslov et al., "Optical-resolution photoacoustic microscopy for in vivo imaging of single capillaries," Opt. Lett. 33(9), 929-931 (2008).

15. S. Hu, K. Maslov, and L. V. Wang, "Second-generation optical-resolution photoacoustic microscopy with improved sensitivity and speed," Opt. Lett. 36(7), 1134-1136 (2011).

16. S. Hu et al., "Functional transcranial brain imaging by optical-resolution photoacoustic microscopy," J. Biomed. Opt. 14(4), 040503 (2009).

17. E. W. Stein, K. Maslov, and L. H. V. Wang, "Noninvasive, in vivo imaging of the mouse brain using photoacoustic microscopy," J. Appl. Phys. 105(10), 102027 (2009).

18. X. D. Wang et al., "Noninvasive laser-induced photoacoustic tomography for structural and functional in vivo imaging of the brain," Nat. Biotechnol. 21(7), 803-806 (2003).

19. Y. Xu and L. H. V. Wang, "Rhesus monkey brain imaging through intact skull with thermoacoustic tomography," IEEE Trans. Ultrason. Ferroelectr. Freq. Control 53(3), 542-548 (2006).

20. T. P. Matthews et al., "Label-free photoacoustic microscopy of peripheral nerves," J. Biomed. Opt. 19(1), 16004 (2014).

21. H. F. Zhang et al., "Functional photoacoustic microscopy for highresolution and noninvasive in vivo imaging," Nat. Biotechnol. 24(7), 848-851 (2006).

22. K. Maslov, G. Stoica, and L. H. V. Wang, "In vivo dark-field reflectionmode photoacoustic microscopy," Opt. Lett. 30(6), 625-627 (2005).

23. C. Li et al., "Real-time photoacoustic tomography of cortical hemodynamics in small animals," J. Biomed. Opt. 15(1), 010509 (2010).

24. Y. Wang et al., "In vivo three-dimensional photoacoustic imaging based on a clinical matrix array ultrasound probe," J. Biomed. Opt. 17(6), 061208 (2012).

25. E. Z. Zhang et al., "In vivo high-resolution 3D photoacoustic imaging of superficial vascular anatomy," Phys. Med. Biol. 54(4), 1035-1046 (2009).

26. J. Laufer et al., "Three-dimensional noninvasive imaging of the vasculature in the mouse brain using a high resolution photoacoustic scanner," Appl. Opt. 48(10), D299-D306 (2009).

27. N. C. Burton et al., "Multispectral opto-acoustic tomography (MSOT) of the brain and glioblastoma characterization," Neuroimage 65, 522528 (2013).

28. R. A. Kruger et al., "Photoacoustic angiography of the breast," Med. Phys. 37(11), 6096-6100 (2010).

29. H. P. Brecht et al., "Whole-body three-dimensional optoacoustic tomography system for small animals," J. Biomed. Opt. 14(6), 064007 (2009).

30. A. Buehler et al., "Video rate optoacoustic tomography of mouse kidney perfusion," Opt. Lett. 35(14), 2475-2477 (2010).

31. M. H. Xu and L. V. Wang, "Analytic explanation of spatial resolution related to bandwidth and detector aperture size in thermoacoustic or photoacoustic reconstruction," Phys. Rev. E 67, 056605 (2003).

32. X. M. Yang and L. V. Wang, "Monkey brain cortex imaging by photoacoustic tomography," J. Biomed. Opt. 13(4), 044009 (2008).

33. R. J. Zemp et al., "Photoacoustic imaging of the microvasculature with a high-frequency ultrasound array transducer," J. Biomed. Opt. 12(1), 010501 (2007).

34. K. H. Song and L. V. Wang, "Deep reflection-mode photoacoustic imaging of biological tissue," J. Biomed. Opt. 12(6), 060503 (2007).

35. W. X. Xing et al., "Integrated optical- and acoustic-resolution photoacoustic microscopy based on an optical fiber bundle," Opt. Lett. 38(1), 52-54 (2013).

36. J. Xia et al., "Wide-field two-dimensional multifocal optical-resolution photoacoustic-computed microscopy," Opt. Lett. 38(24), 5236-5239 (2013).

37. W. Xing et al., "Integrated optical- and acoustic-resolution photoacoustic microscopy based on an optical fiber bundle," Opt. Lett. 38(1), 52-54 (2013). 
38. P. C. Beard, "Photoacoustic imaging for characterising the structure and function of vascular networks," J. Acoust. Soc. Am. 123(5), 3369-3369 (2008).

39. E. W. Stein, K. Maslov, and L. H. V. Wang, "Noninvasive, in vivo imaging of blood-oxygenation dynamics within the mouse brain using photoacoustic microscopy," J. Biomed. Opt. 14(2), 020502 (2009).

40. J. Xia et al., "Calibration-free quantification of absolute oxygen saturation based on the dynamics of photoacoustic signals," Opt. Lett. 38(15), 2800-2803 (2013).

41. L. D. Liao et al., "Imaging brain hemodynamic changes during rat forepaw electrical stimulation using functional photoacoustic microscopy," Neuroimage 52(2), 562-570 (2010).

42. L. D. Liao et al., "Transcranial imaging of functional cerebral hemodynamic changes in single blood vessels using in vivo photoacoustic microscopy," J. Cereb. Blood Flow Metab. 32(6), 938-951 (2012).

43. Z. J. Guo, S. Hu, and L. H. V. Wang, "Calibration-free absolute quantification of optical absorption coefficients using acoustic spectra in 3D photoacoustic microscopy of biological tissue," Opt. Lett. 35(12), 2067-2069 (2010).

44. Z. Guo et al., "Quantitative photoacoustic microscopy of optical absorption coefficients from acoustic spectra in the optical diffusive regime," J. Biomed. Opt. 17(6), 066011 (2012).

45. M. E. Raichle and M. A. Mintun, "Brain work and brain imaging," Annu. Rev. Neurosci. 29, 449-476 (2006).

46. D. Hanahan and R. A. Weinberg, "Hallmarks of cancer: the next generation," Cell 144(5), 646-674 (2011).

47. Z. L. Deng et al., "In vivo imaging of hemodynamics and oxygen metabolism in acute focal cerebral ischemic rats with laser speckle imaging and functional photoacoustic microscopy," J. Biomed. Opt. 17(8), 081415 (2012).

48. L. D. Wang, K. Maslov, and L. H. V. Wang, "Single-cell label-free photoacoustic flowoxigraphy in vivo," Proc. Natl. Acad. Sci. U. S. A. 110(15), 5759-5764 (2013).

49. H. A. MacKenzie et al., "Advances in photoacoustic noninvasive glucose testing," Clin. Chem. 45(9), 1587-1595 (1999).

50. J. Yao et al., "Noninvasive photoacoustic computed tomography of mouse brain metabolism in vivo," Neuroimage 64, 257-66 (2013).

51. Z. Cheng et al., "Near-infrared fluorescent deoxyglucose analogue for tumor optical imaging in cell culture and living mice," Bioconjugate Chem. 17(3), 662-669 (2006).

52. J. C. Pickup et al., "Fluorescence-based glucose sensors," Biosens. Bioelectron. 20(12), 2555-2565 (2005).

53. K. M. Ye and J. S. Schultz, "Genetic engineering of an allosterically based glucose indicator protein for continuous glucose monitoring by fluorescence resonance energy transfer," Anal. Chem. 75(14), 3451-3459 (2003).

54. M. L. Scholvinck et al., "Neural basis of global resting-state fMRI activity," Proc. Natl. Acad. Sci. U. S. A. 107(22), 10238-10243 (2010).

55. D. A. Gusnard and M. E. Raichle, "Searching for a baseline: functional imaging and the resting human brain," Nat. Rev. Neurosci. 2(10), 685694 (2001).

56. M. Nasiriavanaki et al., "High-resolution photoacoustic tomography of resting-state functional connectivity in the mouse brain," Proc. Natl. Acad. Sci. U. S. A. 111(1), 21-26 (2014).

57. V. Tsytsarev et al., "Photoacoustic microscopy of microvascular responses to cortical electrical stimulation," J. Biomed. Opt. 16(7), 076002 (2011).

58. J. Jo and X. Yang, "Functional photoacoustic imaging to observe regional brain activation induced by cocaine hydrochloride," J. Biomed. Opt. 16(9), 090506 (2011).

59. M. F. Lythgoe, N. R. Sibson, and N. G. Harris, "Neuroimaging of animal models of brain disease," Br. Med. Bull. 65(1), 235-257 (2003).

60. F. J. van der Staay, S. S. Arndt, and R. E. Nordquist, "Evaluation of animal models of neurobehavioral disorders," Behav. Brain Funct. 5, 11 (2009).

61. M. L. Li et al., "Simultaneous molecular and hypoxia imaging of brain tumors in vivo using spectroscopic photoacoustic tomography," Proc. IEEE 96(3), 481-489 (2008).

62. J. Staley et al., "Growth of melanoma brain tumors monitored by photoacoustic microscopy," J. Biomed. Opt. 15(4), 040510 (2010).
63. Z. Xu, Q. I. Zhu, and L. H. V. Wang, "In vivo photoacoustic tomography of mouse cerebral edema induced by cold injury," J. Biomed. Opt. 16(6), 066020 (2011).

64. S. Hu et al., "Optical-resolution photoacoustic microscopy of ischemic stroke," Proc. SPIE 7899, 789906 (2011).

65. Q. Z. Zhang et al., "Non-invasive imaging of epileptic seizures in vivo using photoacoustic tomography," Phys. Med. Biol. 53(7), 1921-1931 (2008).

66. V. Tsytsarev et al., "Photoacoustic and optical coherence tomography of epilepsy with high temporal and spatial resolution and dual optical contrasts," J. Neurosci. Methods 216(2), 142-145 (2013).

67. Y. Y. Liu et al., "Assessing the effects of norepinephrine on single cerebral microvessels using optical-resolution photoacoustic microscope," J. Biomed. Opt. 18(7), 076007 (2013).

68. S. H. Yang et al., "Noninvasive monitoring of traumatic brain injury and post-traumatic rehabilitation with laser-induced photoacoustic imaging," Appl. Phys. Lett. 90(24), 243902 (2007).

69. K. Firouzi and N. Saffari, "Numerical modeling of photoacoustic imaging of brain tumors," J. Acoust. Soc. Am. 129(4), 2537-2611 (2011).

70. E. Guevara et al., "Imaging of an inflammatory injury in the newborn rat brain with photoacoustic tomography," PLoS One 8(12), e83045 (2013).

71. H. McIlwain and H. S. Bachelard, Biochemistry and the Central Nervous System, 5th ed., p. 660, Churchill Livingstone, Edinburgh; New York (1985).

72. Z. Xu, C. H. Li, and L. V. Wang, "Photoacoustic tomography of water in phantoms and tissue," J. Biomed. Opt. 15(3), 036019 (2010).

73. D. Razansky, J. Baeten, and V. Ntziachristos, "Sensitivity of molecular target detection by multispectral optoacoustic tomography (MSOT)," Med. Phys. 36(3), 939-945 (2009).

74. A. M. Winkler, K. Maslov, and L. V. Wang, "Noise-equivalent sensitivity of photoacoustics," J. Biomed. Opt. 18(9), 097003 (2013).

75. J. Laufer et al., "In vivo preclinical photoacoustic imaging of tumor vasculature development and therapy," J. Biomed. Opt. 17(5), 056016 (2012).

76. J. Glatz et al., "Blind source unmixing in multi-spectral optoacoustic tomography," Opt. Express 19(4), 3175-3184 (2011).

77. K. E. Wilson, T. Y. Wang, and J. K. Willmann, "Acoustic and photoacoustic molecular imaging of cancer," J. Nucl. Med. 54(11), 18511854 (2013).

78. A. de la Zerda et al., "Advanced contrast nanoagents for photoacoustic molecular imaging, cytometry, blood test and photothermal theranostics," Contrast Media Mol. Imaging 6(5), 346-369 (2011).

79. X. M. Yang et al., "Nanoparticles for photoacoustic imaging," Wiley Interdiscip. Rev.: Comput. Mol. Sci. 1(4), 360-368 (2009).

80. V. Ntziachristos and D. Razansky, "Molecular imaging by means of multispectral optoacoustic tomography (MSOT)," Chem. Rev. 110(5), 2783-2794 (2010).

81. D. Razansky, A. Buehler, and V. Ntziachristos, "Volumetric real-time multispectral optoacoustic tomography of biomarkers," Nat. Protocols 6(8), 1121-1129 (2011).

82. G. P. Luke, D. Yeager, and S. Y. Emelianov, "Biomedical applications of photoacoustic imaging with exogenous contrast agents," Ann. Biomed. Eng. 40(2), 422-437 (2012).

83. K. K. Jain, "Nanobiotechnology-based strategies for crossing the blood-brain barrier," Nanomedicine 7(8), 1225-1233 (2012).

84. G. A. Silva, "Nanotechnology approaches to crossing the blood-brain barrier and drug delivery to the CNS," BMC Neurosci. 9, S4 (2008).

85. P. Ballabh, A. Braun, and M. Nedergaard, "The blood-brain barrier: an overview: structure, regulation, and clinical implications," Neurobiol. Dis. 16(1), 1-13 (2004).

86. R. A. Sheth, L. Josephson, and U. Mahmood, "Evaluation and clinically relevant applications of a fluorescent imaging analog to fluorodeoxyglucose positron emission tomography," J. Biomed. Opt. 14(6), 064014 (2009).

87. R. G. O'Neil, L. Wu, and N. Mullani, "Uptake of a fluorescent deoxyglucose analog (2-NBDG) in tumor cells," Mol. Imaging Biol. 7(6), 388-392 (2005).

88. M. C. Pilatou et al., "Photoacoustic imaging of brain perfusion on albino rats by using evans blue as contrast agent," Arch. Physiol. Biochem. 111(4), 389-397 (2003). 
89. M. R. Chatni et al., "Tumor glucose metabolism imaged in vivo in small animals with whole-body photoacoustic computed tomography," J. Biomed. Opt. 17(7), 076012 (2012).

90. M. F. Kircher et al., "A brain tumor molecular imaging strategy using a new triple-modality MRI-photoacoustic-Raman nanoparticle," Nat. Med. 18(5), 829-835 (2012).

91. P. H. Wang et al., "Gold-nanorod contrast-enhanced photoacoustic micro-imaging of focused-ultrasound induced blood-brain-barrier opening in a rat model," J. Biomed. Opt. 17(6), 061222 (2012).

92. M. Wang, J. Etu, and S. Joshi, "Enhanced disruption of the blood brain barrier by intracarotid mannitol injection during transient cerebral hypoperfusion in rabbits,' J. Neurosurg. Anesthesiol. 19(4), 249-256 (2007).

93. L. S. Bouchard et al., "Picomolar sensitivity MRI and photoacoustic imaging of cobalt nanoparticles," Proc. Natl. Acad. Sci. U. S. A. 106(11), 4085-4089 (2009).

94. J. Levi et al., "Design, synthesis, and imaging of an activatable photoacoustic probe," J. Am. Chem. Soc. 132(32), 11264-11269 (2010).

95. W. Lu et al., "Photoacoustic imaging of living mouse brain vasculature using hollow gold nanospheres," Biomaterials 31(9), 2617-2626 (2010).

96. Y. W. Wang et al., "Photoacoustic tomography of a nanoshell contrast agent in the in vivo rat brain," Nano Lett. 4(9), 1689-1692 (2004).

97. A. Ray et al., "Targeted blue nanoparticles as photoacoustic contrast agent for brain tumor delineation," Nano Res. 4(11), 1163-1173 (2011).

98. J. Levi et al., "Molecular photoacoustic imaging of follicular thyroid carcinoma," Clin. Cancer Res. 19(6), 1494-1502 (2013).

99. S. Zackrisson, S. M. van de Ven, and S. S. Gambhir, "Light in and sound out: emerging translational strategies for photoacoustic imaging," Cancer Res. 74(4), 979-1004 (2014).

100. A. De la Zerda and S. S. Gambhir, "Drug delivery-keeping tabs on nanocarriers," Nat. Nanotechnol. 2(12), 745-746 (2007).

101. K. Pancholi, "A review of imaging methods for measuring drug release at nanometre scale: a case for drug delivery systems," Expert Opin. Drug Delivery 9(2), 203-218 (2012).

102. C. Kim, C. Favazza, and L. H. V. Wang, "In vivo photoacoustic tomography of chemicals: high-resolution functional and molecular optical imaging at new depths," Chem. Rev. 110(5), 2756-2782 (2010).

103. K. H. Song et al., "Near-infrared gold nanocages as a new class of tracers for photoacoustic sentinel lymph node mapping on a rat model," Nano Lett. 9(1), 183-188 (2009).

104. H. Maeda, "Macromolecular therapeutics in cancer treatment: the EPR effect and beyond," J. Controlled Release 164(2), 138-144 (2012).

105. D. Razansky et al., "Multispectral opto-acoustic tomography of deepseated fluorescent proteins in vivo," Nat. Photonics 3(7), 412-417 (2009).

106. G. S. Filonov et al., "Deep-tissue photoacoustic tomography of a genetically encoded near-infrared fluorescent probe," Angew. Chem. Int. Ed. 51(6), 1448-1451 (2012).

107. J. Laufer et al., "In vitro characterization of genetically expressed absorbing proteins using photoacoustic spectroscopy," Biomed. Opt. Express 4(11), 2477-2490 (2013).

108. N. C. Deliolanis et al., "Deep-tissue reporter-gene imaging with fluorescence and optoacoustic tomography: a performance overview," Mol. Imaging Biol. (2014).
109. D. Razansky, C. Vinegoni, and V. Ntziachristos, "Multispectral photoacoustic imaging of fluorochromes in small animals," Opt. Lett. 32(19), 2891-2893 (2007).

110. L. Li et al., "Photoacoustic imaging of lacZ gene expression in vivo," J. Biomed. Opt. 12(2), 020504 (2007).

111. A. Krumholz et al., "Photoacoustic microscopy of tyrosinase reporter gene in vivo," J. Biomed. Opt. 16(8), 080503 (2011).

112. R. J. Paproski et al., "Tyrosinase as a dual reporter gene for both photoacoustic and magnetic resonance imaging," Biomed. Opt. Express 2(4), 771-780 (2011).

113. J. Laufer et al., "In vivo photoacoustic imaging of tyrosinase expressing tumours in mice," Proc. SPIE 8223, 82230M (2012).

114. C. X. Qin et al., "Tyrosinase as a multifunctional reporter gene for photoacoustic/MRI/PET triple modality molecular imaging," Sci. Rep. 3, 1490 (2013).

115. M. Firbank et al., "Measurement of the optical-properties of the skull in the wavelength range 650-950 Nm," Phys. Med. Biol. 38(4), 503510 (1993).

116. L. M. Nie et al., "Photoacoustic tomography through a whole adult human skull with a photon recycler," J. Biomed. Opt. 17(11), 110506 (2012).

117. H. Azhari, Wiley InterScience (Online service), Basics of Biomedical Ultrasound for Engineers, Wiley, IEEE, Hoboken, New Jersey (2010).

118. F. J. Fry and J. E. Barger, "Acoustical properties of the human skull," J. Acoust. Soc. Am. 63(5), 1576-1590 (1978).

119. C. Huang et al., "Aberration correction for transcranial photoacoustic tomography of primates employing adjunct image data," J. Biomed. Opt. 17(6), 066016 (2012).

120. X. Wang et al., "Noninvasive photoacoustic angiography of animal brains in vivo with near-infrared light and an optical contrast agent," Opt. Lett. 29(7), 730-732 (2004).

121. Y.-L. He et al., "Measurement of blood volume using indocyanine green measured with pulse-spectrophotometry: its reproducibility and reliability," Crit. Care Med. 26(8), 1446-1451 (1998).

122. J. Xia et al., "Enhancement of photoacoustic tomography by ultrasonic computed tomography based on optical excitation of elements of a full-ring transducer array," Opt. Lett. 38(16), 3140-3143 (2013).

123. B. A. Flusberg et al., "In vivo brain imaging using a portable 3.9 gram two-photon fluorescence microendoscope," Opt. Lett. 30(17), 22722274 (2005).

124. F. Helmchen et al., "A miniature head-mounted two-photon microscope: high-resolution brain imaging in freely moving animals," Neuron 31(6), 903-912 (2001).

125. D. A. Dombeck et al., "Imaging large-scale neural activity with cellular resolution in awake, mobile mice," Neuron 56(1), 43-57 (2007).

126. E. M. C. Hillman, "Optical brain imaging in vivo: techniques and applications from animal to man," J. Biomed. Opt. 12(5), 051402 (2007).

Junjie Yao is currently a postdoctoral research associate at Washington University in St. Louis.

Lihong V. Wang holds the Gene K. Beare Distinguished Professorship at Washington University in St. Louis. 